\title{
Gold/Titania Nanorod Assembled Urchin-Like Photocatalysts with an Enhanced Hydrogen Generation by Photocatalytic Biomass Reforming
}

Cai Shi, ${ }^{1, \#}$ Weijie Yuan, ${ }^{1, \#}$ Keqi Qu, ${ }^{1}$ Junming Shi, ${ }^{1}$ Malin Eqi, ${ }^{1}$ Xushen Tan, ${ }^{1}$ Zhanhua Huang,,${ }^{1, *}$ Felipe Gándara,,${ }^{2, *}$ Duo Pan, ${ }^{3,5}$ Nithesh Naik, ${ }^{4}$ Yixiang Zhang ${ }^{1}$ and Zhanhu Guo ${ }^{5,}$

\begin{abstract}
Herein, we successfully constructed $\mathrm{Au} /$ hierarchical urchin-like $\mathrm{TiO}_{2}$ architectures $(\mathrm{Au} / \mathrm{HT})$ photocatalysts, which were assembled by crystalline nanorods by one-step solvothermal method under mild conditions. The as-prepared samples showed an outstandingly renewable $\mathrm{H}_{2}$ evolution by the photocatalytic glucose reforming. $\mathrm{Au} / \mathrm{HT}$ revealed a high $\mathrm{H}_{2}$ evolution rate of $616.23 \mu \mathrm{mol} \mathrm{h}^{-1} \mathrm{~g}^{-1}$ under visible-light irradiation at low temperatures without any additional Pt co-catalyst, which was about 46.23 and 15.81 times than that of P25 and HT, since the (one-dimensional) 1D nanorods could effectively accelerate electron transfer and restrain the recombination of photogenerated carriers, meanwhile, (three-dimensional) 3D hierarchical architectures could improve the light capturing capability of $\mathrm{HT}$, and surface-plasmon resonance (SPR) effect of $\mathrm{Au}$ nanoparticles, which further improved the separation efficiency of photogenerated charges, extended the visible light absorption, and accelerated the $\mathrm{H}_{2}$ generation. This work offers a potential way to produce sustainable $\mathrm{H}_{2}$ from environmentally renewable biomass materials using solar energy.
\end{abstract}

Keywords: Au/hierarchical urchin-like $\mathrm{TiO}_{2}$ architectures; Biomass; Photoreforming; $\mathrm{H}_{2}$ evolution; Photocatalysis.

Received: 27 March 2021; Accepted: 05 Jun 2021.

Article type: Research article.

\section{Introduction}

Over the past years, it has become evident that there is an urgent societal need to develop and exploit efficient and clean energy sources alternative to the traditional fossil fuels. ${ }^{[1-6]}$ The use of hydrogen $\left(\mathrm{H}_{2}\right)$ as an energy vector offers great promise, as its combustion does not cause any pollution. ${ }^{[7-9]}$ The generation of $\mathrm{H}_{2}$ from renewable sources, including solar energy, water and biomass, which is a most appealing approach to reach the ultimate goal of a zero emission and carbon neutral economy. ${ }^{[10-12]}$ In this context, $\mathrm{H}_{2}$ generation through photocatalytic water splitting has become a theme of

\footnotetext{
${ }^{1}$ Key Laboratory of Bio-based Material Science and Technology,

Northeast Forestry University, Ministry of Education, Harbin 150040, China.

${ }^{2}$ Materials Science Factory, Instituto de Ciencia de Materiales de Madrid (ICMM)-Consejo Superior de Investigaciones Científicas (CSIC), C/Sor Juana Inés de la Cruz, 3, Madrid 28049, Spain.

${ }^{3}$ Key Laboratory of Materials Processing and Mold (Zhengzhou University), Ministry of Education, National Engineering Research Center for Advanced Polymer Processing Technology, Zhengzhou University, Zhengzhou, 450001, China.
}

extensive research. ${ }^{[13-19]}$ However, in general, the addition of sacrificial electron donors (such as methanol, organic acids, or amines, among others) is required, ${ }^{[20-23]}$ which inevitably results in a waste of valuable chemicals. Biomass is the most abundant renewable resource on earth, and it accounts for a large part of the energy source for mankind. ${ }^{24-30]}$ Moreover, natural biomass or biomass-derived substances are quite suitable precursors for the photocatalytic $\mathrm{H}_{2}$ evolution. Glucose, a kind of simple structure sugar aldoses, is one of the most significant building block in the carbohydrate family and it has a high $\mathrm{H} / \mathrm{C}$ ratio. It can be easily obtained from renewable biomass, making it ideal for photocatalytic $\mathrm{H}_{2}$ generation. ${ }^{[3]}$ In the process of photocatalytic glucose reforming for $\mathrm{H}_{2}$ evolution, electron and hole pairs $\left(\mathrm{e}^{-} / \mathrm{h}^{+}\right)$are generated upon light irradiation with energy higher than the bandgap of the semiconducting photocatalysts. The $\mathrm{e}^{-}$in conduction band (CB) would reduce water to generate $\mathrm{H}_{2}$, while the $\mathrm{h}^{+}$in the valence band (VB) may be captured by $\mathrm{H}_{2} \mathrm{O}$ or $\mathrm{OH}^{-}$in aqueous solution to form highly oxidizing hydroxyl radicals $(\bullet \mathrm{OH})$. These strong oxidizing substances $(\bullet \mathrm{OH}$ and $\mathrm{h}^{+}$) would oxidize glucose to produce value-added chemicals. ${ }^{[32,33]}$ 
$\mathrm{TiO}_{2}$ remains as the most widely studied semiconductor photocatalyst, and the mechanism of photocatalytic reforming process has been extensively researched. ${ }^{[31,34-41]}$ Although $\mathrm{TiO}_{2}$ has been extensively researched as a photocatalyst, its further application in the field of photocatalytic $\mathrm{H}_{2}$ generation has been hampered by its inherent shortcomings, notably the limited light absorption to only UV region and the rapid recombination of photogenerated carriers. ${ }^{[42,43]}$ With an improved photocatalytic performance, the crystalline hierarchical $\mathrm{TiO}_{2}$ (HT) nanorods effectively accelerated the $\mathrm{e}$ transfer rate and restrained the recombination of photogenerated carriers. ${ }^{[44-46]}$ Moreover, the modification of the HT with noble metal nanostructures $(\mathrm{Au})$ is a promising method to further extend the visible light absorption of $\mathrm{TiO}_{2}$ and improve its photocatalytic efficiency. ${ }^{[47]}$ The unique surface-plasmon resonance (SPR) effect of Au offers a large local electric field and tunable resonance wavelength, which further improve the separation efficiency of photogenerated charges, extend the visible light absorption, and efficiently enhance the photocatalytic activity. ${ }^{[43,48,49]}$

In this study, aiming to facilitate the utilization of renewable energy such as biomass and solar energy, we report herein the synthesis of Au/HT photocatalysts by one-step solvothermal method under mild conditions. SPR effect was observed for $\mathrm{H}_{2}$ generation. The prepared Au/HT photocatalysts exhibited significantly enhanced visible light absorption and $\mathrm{e}^{-} / \mathrm{h}^{+}$separation efficiency, and the photocatalytic $\mathrm{H}_{2}$ evolution activity from glucose reforming was also improved prominently. The highest $\mathrm{H}_{2}$ production rate of one of the materials here reported (HTA4) reached $616.23 \mu \mathrm{mol} \mathrm{h}^{-1} \mathrm{~g}^{-1}$, which was 46.23 times higher than that of commercial $\mathrm{TiO}_{2}$ photocatalyst (P25). Finally, the possible mechanism with the use of Au/HT photocatalysts under visible light irradiation is proposed, including the promoted charge transfer and the enhanced light absorption caused by the 3D hierarchical architectures composed of nanorods and the SPR effect of Au.

\section{Experimental part}

\subsection{Synthesis of photocatalysts}

HT were prepared according to our previous work by a solvothermal method. ${ }^{[42]}$ Firstly, $0.5 \mathrm{~mL}$ of titanium tetrachloride was added to $3 \mathrm{~mL}$ of acetone to constitute a mixture, which was ultrasonicated for $30 \mathrm{~min}$, then placed into a Teflon-lined autoclave containing deionized water $(3 \mathrm{~mL})$

\footnotetext{
4 Department of Mechanical \& Manufacturing Engineering, Manipal Institute of Technology, Manipal Academy of Higher Education, Manipal-576104, Karnataka, India.

5 Integrated Composites Laboratory (ICL), Department of Chemical and Bimolecular Engineering, University of Tennessee, Knoxville, TN, 37996, USA.

\# means co-first author.

*Email: huangzh1975@163.com (Z. Huang), zguo10@utk.edu (Z. Guo)
}

and reacted at $135{ }^{\circ} \mathrm{C}$ for $48 \mathrm{~h}$. After the reaction, the obtained HT was centrifuged and washed with ethanol and water, dried at room temperature. Then, the Au/HT photocatalysts were obtained using a hydrothermal method. $0.2 \mathrm{~g}$ HT were mixed with different volumes of a $0.01 \mathrm{M} \mathrm{HAuCl}_{4}$ solution $(0.24$, $0.49,1.46,1.94$, and $2.43 \mathrm{~mL}$ ) in a mixed solution of $20 \mathrm{~mL}$ $\mathrm{H}_{2} \mathrm{O}$ and $10 \mathrm{~mL}$ of ethanol, and stirred for 30 minutes, then transferred to a Teflon-lined autoclave, and heated at $140{ }^{\circ} \mathrm{C}$ for $4 \mathrm{~h}$. The resulting Au/HT photocatalysts with different initial Au contents $(0,1,3,4$, and $5 \mathrm{wt} \%)$ were washed and centrifuged with DI water and then dried under vacuum overnight. The samples were labeled as HTAx, with $\mathrm{x}=0,1$, 3,4 , and $5 \mathrm{wt} \%$, respectively.

\subsection{Photocatalytic $\mathrm{H}_{2}$ evolution activity}

Photocatalytic $\mathrm{H}_{2}$ evolution from water splitting. The $\mathrm{H}_{2}$ production test were executed in an airtight Pyrex container $(350 \mathrm{~mL})$, which had a gas discharge and circulation photocatalytic system under visible light irradiated with a 300W Xenon lamp (Hxuv300 Visref, Beijing CEJ Tech. Co., Ltd., Beijing, China) on the top. In a typical representative experiment, the as-prepared photocatalyst $(50 \mathrm{mg})$ was suspended into $100 \mathrm{~mL}$ aqueous solution containing $10 \%(\mathrm{v} / \mathrm{v})$ triethanolamine (TEOA) as sacrificial $\mathrm{e}^{-}$donor, and magnetically stirred. No cocatalyst was placed in the photocatalytic system. The reactor was degassed $30 \mathrm{~min}$ by a vacuum pump to remove air before irradiation and make sure that the $\mathrm{H}_{2}$ production reaction was conducted under vacuum conditions. The reaction temperature was kept at $279 \mathrm{~K}$ by circulating cooling water. Subsequently, the generated $\mathrm{H}_{2}$ was collected every $30 \mathrm{~min}$ and automatically quantified by gas chromatography (GC-7920, Beijing CEJ Tech. Co., Ltd., Beijing, China) with a thermal conductivity detector (TCD), and $\mathrm{N}_{2}$ was used as carrier gas. Each photocatalytic reaction lasted for $4 \mathrm{~h}$.

Photocatalytic $\mathrm{H}_{2}$ evolution from biomass reforming. The $\mathrm{H}_{2}$ production device used in this process was the same as the $\mathrm{H}_{2}$ evolution from water decomposition, and the environmental conditions of the reaction were also the same. The difference was that the $100 \mathrm{~mL}$ solution containing $10 \%$ $(\mathrm{v} / \mathrm{v})$ TEOA was replaced by a glucose aqueous solution (100 $\mathrm{mL}$ ) with a concentration of $1000 \mathrm{mg} \mathrm{L}^{-1}$. The slurry was magnetically stirred for $2 \mathrm{~h}$ under dark conditions to realize an adsorption-desorption equilibrium of glucose on the surface of photocatalyst. The produced $\mathrm{H}_{2}$ was automatically quantitatively detected by gas chromatography (GC-7920, Beijing CEJ Tech. Co., Ltd., Beijing, China) with a thermal conductivity detector (TCD) every $30 \mathrm{~min}$, and $\mathrm{N}_{2}$ was used as carrier gas.

\subsection{Photoelectrochemical test}

Electrochemical impedance spectra (EIS) and photocurrent transient response (I-t) were measured in a three-electrode system [counter electrode: Pt plate; reference electrode: $\mathrm{Ag} / \mathrm{AgCl}$; working electrode: fluorine-doped tin oxide (FTO) 
glass with photocatalyst] on an electrochemical workstation (CHI660E, Shanghai, China). $\mathrm{Na}_{2} \mathrm{SO}_{4}$ aqueous solution (0.50 $\mathrm{M})$ was used as the electrolyte. The light irradiation was supplied by a $300-\mathrm{W}$ Xenon lamp source $(\lambda \geq 420 \mathrm{~nm})$. The preparation of working electrode was conducted as follows: 2 $\mathrm{mg}$ of the pulverous sample was placed in $1 \mathrm{~mL}$ of ethanol, ultrasonicated for $30 \mathrm{~min}$, then nafion solution $(10 \mu \mathrm{L})$ was added and ultrasonicated for another $10 \mathrm{~min}$ to form a slurry. The slurry $(200 \mu \mathrm{L})$ was dipped onto the surface of FTO substrate $(1 \mathrm{~cm} \times 1 \mathrm{~cm})$ and baked with an infrared lamp. The resulting photocatalyst-coated FTO was used as the working electrode for the subsequent EIS and I-t tests.

\section{Results and its discussions}

\subsection{Characterization of catalysts}

The microstructure and morphology of HT were confirmed by field emission scanning electron microscopy (FESEM) and transmission electron microscopy (TEM), as depicted in Fig. 1. Figs. 1a-c shows the FESEM images of synthesized HT. Nearly spherical aggregates with a diameter in the scope of 5 to $7 \mu \mathrm{m}$ are observed and are all formed by many radially grown 1D crystalline nanorods. The TEM images, shown in Figs. 1d and e, further illustrate how the structure of HT is formed by the aggregation of $\mathrm{TiO}_{2}$ crystalline nanorods. The HRTEM (Fig. 1f) reveals that the individual nanorods with 38 $\mathrm{nm}$ in width are highly crystalline, and the measured corresponding lattice spacing of $0.328 \mathrm{~nm}$ corresponds to the (110) crystal plane of rutile $\mathrm{TiO}_{2}$. The selected area electron diffraction (SAED) pattern presented in Fig. If (inset) shows "single-crystal-like" diffraction spots. ${ }^{[50,51]}$ Figs. 1g-h shows the TEM images of HTA4 sample (which is the one with the optimal photocatalytic performance, as discussed below). The images clearly demonstrate that the $\mathrm{Au}$ nanoparticles are successfully loaded on the $\mathrm{TiO}_{2}$ nanorods, and that they are relatively spherical, with the diameter size ranging from 59-82 $\mathrm{nm}$ (the red dotted line). Moreover, the HRTEM (Fig. 1i) shows a well-contacted interface between Au nanoparticles and $\mathrm{TiO}_{2}$ nanorods, which should be beneficial to facilitate $\mathrm{e}^{-}$ transfer from excited Au nanoparticles to HT. The measured lattice fringe spacings of 0.235 and $0.328 \mathrm{~nm}$ are in accordance with $\mathrm{Au}(111)$ and rutile $\mathrm{TiO}_{2}$ (110) lattice planes. ${ }^{[52,53]}$ The TEM and corresponding elemental mapping (Fig. 1j) show the corresponding elemental distribution of HTA4, which confirm the presence of $\mathrm{Ti}, \mathrm{O}$, and $\mathrm{Au}$ elements. A chemical analysis by ICP-OES indicated that the Au loading in HTA4 was $2.56 \%$ $\mathrm{Au}$ with respect to HT. These results, along with the XPS analysis discussed below, demonstrate the successful deposition of Au nanoparticles on the HT surface.

The powder X-ray diffraction (PXRD) patterns of assynthesized HT and HTAx photocatalysts with different Au contents (1-5 wt\%) are shown in Fig. 2a, which further characterize the phase composition and crystallinity. As we reported earlier, ${ }^{[42]}$ the diffraction peaks of $\mathrm{HT}$ at $2 \theta=27.35^{\circ}$, $35.86^{\circ}, 41.05^{\circ}, 43.83^{\circ}, 54.11^{\circ}, 56.42^{\circ}, 63.82^{\circ}$, and $69.57^{\circ}$ are indexed to the (110), (101), (111), (210), (211), (220), (220), and (301) planes of rutile $\mathrm{TiO}_{2},{ }^{[54]}$ while the diffraction peaks located at $2 \theta=25.22^{\circ}, 47.98^{\circ}, 62.63^{\circ}, 68.73^{\circ}$, and $82.26^{\circ}$ belong to the anatase $\mathrm{TiO}_{2}(101),(200),(204),(116)$, and (224)
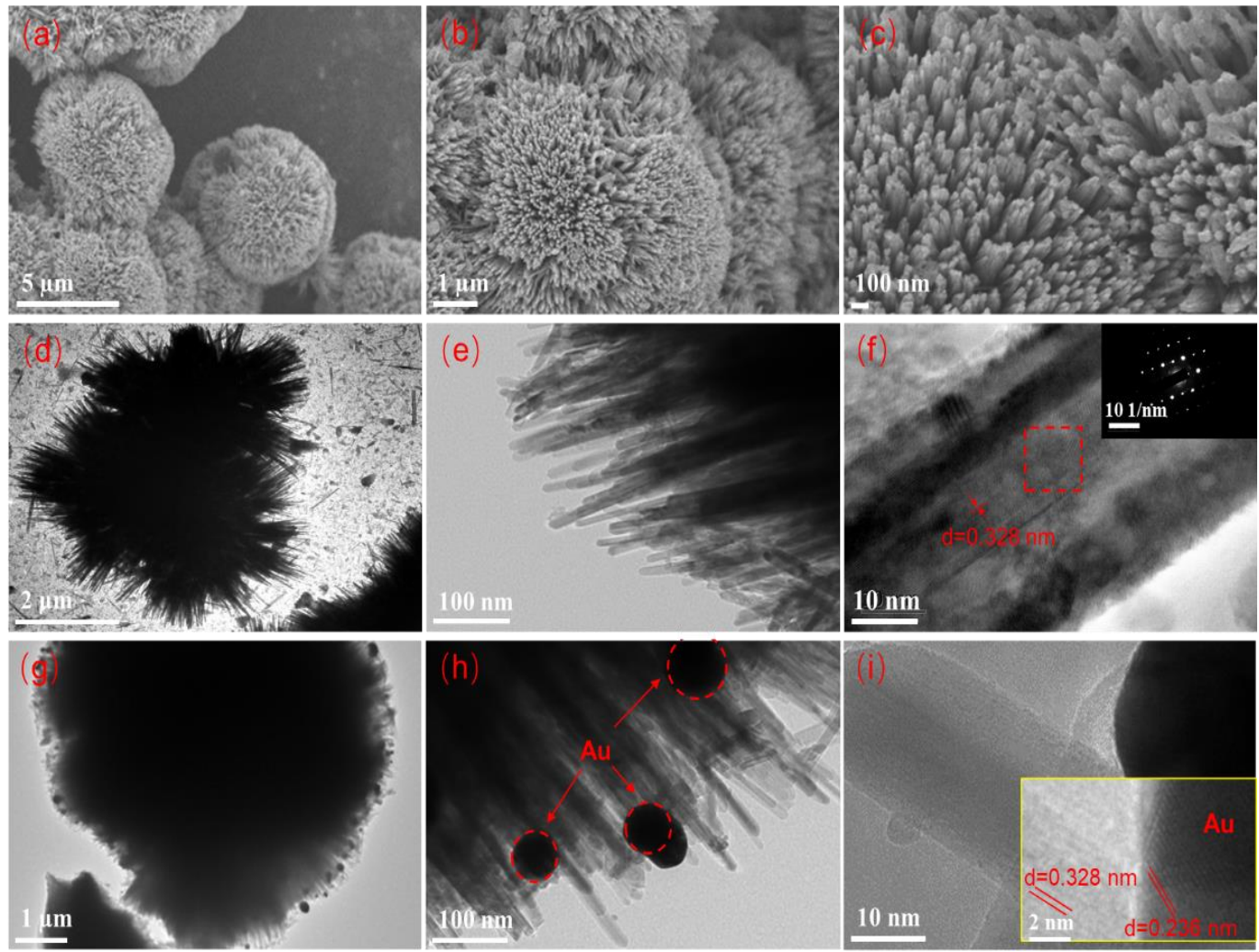

Fig. 1 (a, b, c) FESEM of HT, (d, e, f) TEM and HRTEM of HT (the SAED taken from the dotted line, Fig. 1f inset), (g, h, i) TEM and HRTEM of HTA4, (j) TEM and the corresponding elemental mapping images of an individual HTA4. 
lattice planes. ${ }^{[23]}$ From the characteristic diffraction peaks of HTAx samples, it is clear that rutile $\mathrm{TiO}_{2}$ is still present, which indicates that the incorporation of $\mathrm{Au}$ nanoparticles did not change the main crystal structure of $\mathrm{TiO}_{2}$. Interestingly, for the HTAx samples, the characteristic diffraction peaks of anatase $\mathrm{TiO}_{2}$ at $2 \theta=25.22^{\circ}$ and $47.98^{\circ}$ disappear, as compared to HT PXRD patterns, which may be attributed to the transformation of part of anatase into rutile phase due to the acidic environment provided by $\mathrm{HAuCl}_{4}$ during the preparation of samples. ${ }^{[54,55]}$ The (111), (200), (220), and (311) diffraction peaks of $\mathrm{Au}$ are observed at $2 \theta=38.18^{\circ}, 44.10^{\circ}, 64.76^{\circ}$, and $77.34^{\circ}$, respectively, ${ }^{[56]}$ with higher intensity as the Au amount increases, as expected. We note that the (200) diffraction peak of $\mathrm{Au}$ overlaps with the (210) of rutile $\mathrm{TiO}_{2}$ in the PXRD pattern of HTAx. However, the clear presence of the rest of characteristic diffraction peaks confirms the presence of the Au nanoparticles, which are successfully loaded on the HT surface. Moreover, these PXRD analysis results agree with those from TEM analysis.

The surface area and pore structure of HT, HTA4, and HTA5 were also analyzed by $\mathrm{N}_{2}$ adsorption-desorption isotherms at $77 \mathrm{~K}$, and pore-size distributions. As depicted in
Fig. 2b, the isotherms of HT, HTA4, and HTA5 show type IV profile with a H3 hysteresis loop, ${ }^{[23]}$ which indicates the presence of mesoporous capillary condensation. The existence of mesopores in the samples is attributed to the interparticle voids generated during the aggregation of the HT nanorods. The BET specific surface areas of HT, HTA4, and HTA5 were calculated to be 31,29 , and $25 \mathrm{~m}^{2} \mathrm{~g}^{-1}$, indicating that the specific surface area of the HTA samples decreases after Au incorporation. And they all exhibited a wide pore size distribution of 2-50 nm (Fig. 2b inset), with an average pore size of 7.39, 7.72, and $9.53 \mathrm{~nm}$ for HT, HTA4, and HTA5, and the cumulative pore volume of HT, HTA4, and HTA5 was 0.07 , 0.07 , and $0.06 \mathrm{~cm}^{3} \mathrm{~g}^{-1}$, respectively. The above data shows that their specific surface area has changed significantly upon the incorporation of gold, which can also be explain the lower photocatalytic $\mathrm{H}_{2}$ production of HTA5 than HTA4. ${ }^{57]}$

To get an insight into the surface chemical state and elemental composition of the as-synthesized HTA4, a comprehensive XPS analysis was performed, as shown in Figs 2c-f. Fig. 2c shows the full-scan XPS spectrum of HTA4, from which it can be clearly seen that the sample contains Ti, O, and $\mathrm{Au}$, as well as the presence of adventitious carbon. The
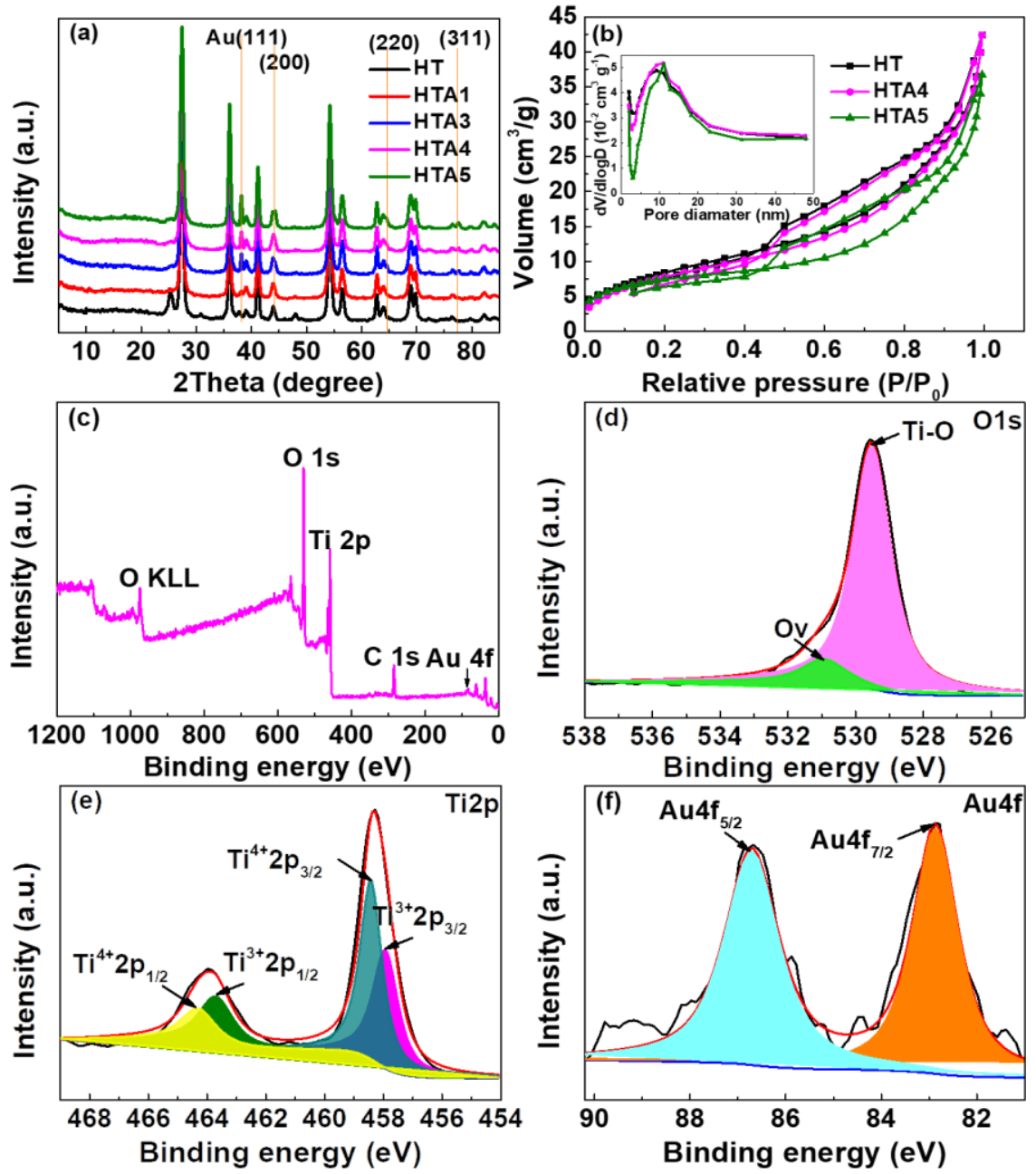

Fig. 2 (a) XRD patterns of HT and HTA with different Au content; (b) Nitrogen adsorption desorption isotherms and the corresponding pore size distribution of HT and HTA4; (c)XPS survey spectrum of HTA4, high-resolution XPS spectra of (d) O1s, (e) Ti2p, (f) Au4f of HTA4. 
acquired high-resolution spectra of $\mathrm{O}, \mathrm{Ti}$, and Au from HTA4 are shown in Figs. 2d-f. Fig. 2d shows the O1s spectra, and two peaks are located at 529.6 and $531.0 \mathrm{eV}$, which belong to the presence of Ti-O peak and oxygen vacancy $(\mathrm{Ov})$. From the high-resolution Ti2p XPS spectrum of HTA4 in Fig. 2e, it can be easily realized the presence of $\mathrm{Ti}^{3+}$ and $\mathrm{Ti}^{4+}$. Thus, the peaks centered at 457.9 and $463.7 \mathrm{eV}$ correspond to Ti2 $\mathrm{p}_{3 / 2}$ and $\mathrm{Ti} 2 \mathrm{p}_{1 / 2}$ of $\mathrm{Ti}^{3+}$, respectively, while those at 458.4 and $464.1 \mathrm{eV}$ arise from the spin states of $2 \mathrm{p}_{3 / 2}$ and $2 \mathrm{p}_{1 / 2}$ of $\mathrm{Ti}^{4+}$ with a 5.7 $\mathrm{eV}$ peak separation. ${ }^{[23,58]}$ It can be inferred from the Ti2p and O1s XPS spectra that the presence of $\mathrm{Ti}^{3+}$ introduces Ov into the composites, which is beneficial for the improvement of its photocatalytic activity. ${ }^{[54,56,59]}$ Fig. 2f displays the XPS spectrum of Au4f, which consists of the peaks centered at 82.9 and $86.7 \mathrm{eV}$, assigned to $\mathrm{Au} 4 \mathrm{f}_{7 / 2}$ and $A u 4 \mathrm{f}_{5 / 2}$, respectively. These binding energy values indicate the formation of metallic $\mathrm{Au}$ phase $\left(\mathrm{Au}^{0}\right){ }^{[60,61]}$ The above XPS results further validate the elemental EDS mapping images, and the successful incorporation of Au nanoparticles.

In general, the capacity of light absorption, and the rapid separation of photo-generated charges and successful transfer to reactive sites on the surface determine the photocatalytic activity of materials. ${ }^{[62]}$ To investigate the behavior of photogenerated carriers, we analyzed the optical and photoelectrochemical properties of the five prepared photocatalysts, as shown in Fig. 3. Fig. 3a shows the UV-vis diffuse reflectance spectra (UV-vis DRS) of HT and various HTAx samples. Interestingly, HT revealed an absorption band at around $424 \mathrm{~nm}$ in the visible light range, which was probably due to the doping of $\mathrm{Ti}^{3+}$ in $\mathrm{HT}^{.63]}$ Compared to HT, the absorption band edges of HTAx samples showed a significant red-shift, while the HTA4 sample demonstrated an absorption edge red shift to $463 \mathrm{~nm}$.

The visible light harvesting capacities of HTAx within 500-600 nm were efficiently heightened with a higher $\mathrm{Au}$ nanoparticle content (SPR effect, the dashed line in Fig. 3a), particularly for the HTA5 sample, ${ }^{[64,65]}$ as expected due to the accumulation of $\mathrm{Au}$ nanoparticles in higher concentrations. The band gap potentials $\left(E_{\mathrm{g}}\right)$ calculated from Tauc plots (Equation 1) of HT and HTA4 (the sample with optimal photocatalytic activity) photocatalysts are 2.92 and $2.68 \mathrm{eV}$ (Fig. 3b), respectively. A lower band gap value is beneficial for visible light absorption and the enhancement of photocatalytic activity, which will be described in detail in the mechanism section.
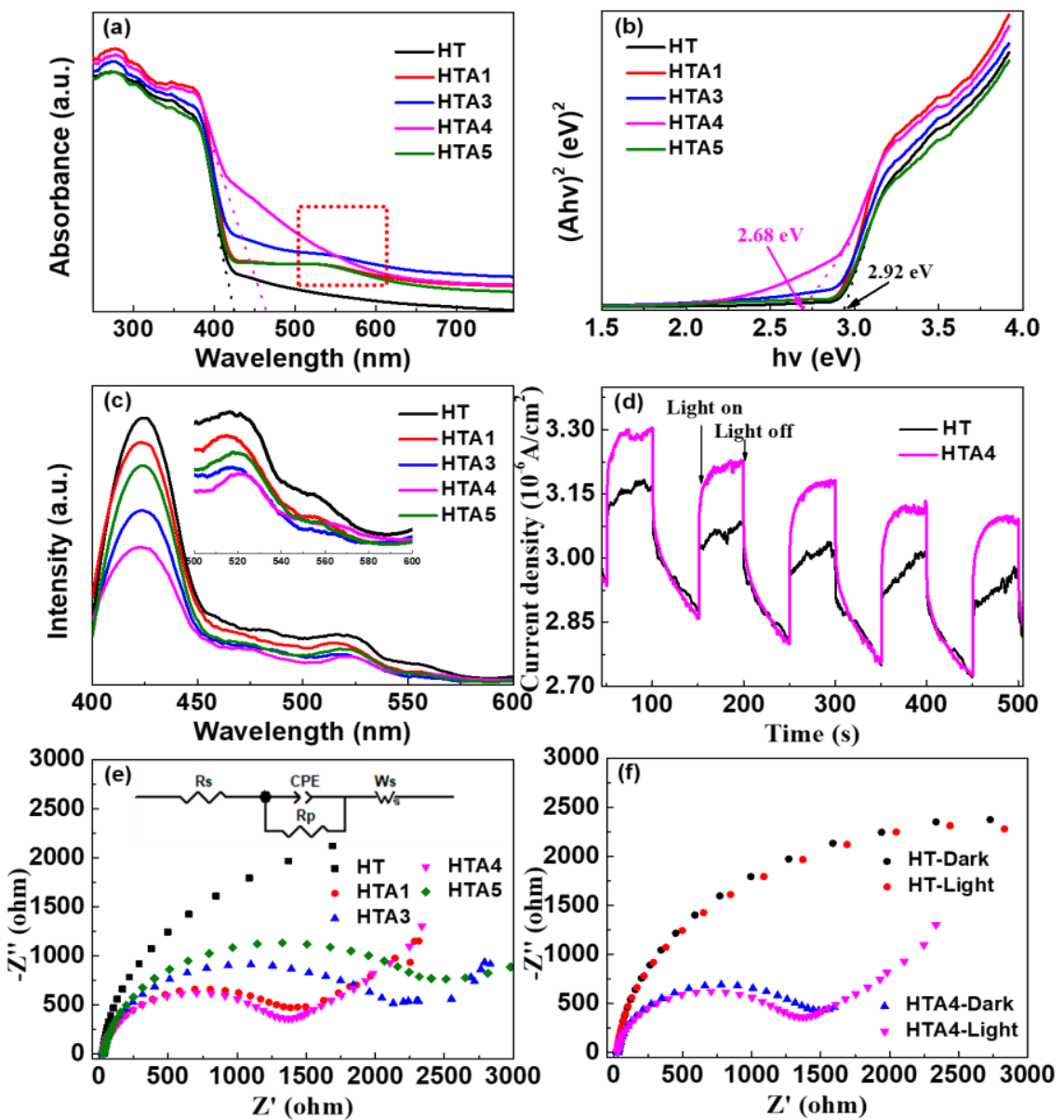

Fig. 3 (a) UV-vis DRS, (b) the corresponding bandgap energy, (c) fluorescence spectra of HT and different HTA samples; (d) transient photocurrent response of HT and HTA4, (e) EIS of HT and different HTA samples under visible-light irradiation and the corresponding equivalent circuit of HTA4 (the inset), (f) EIS of HT and HTA4 in the dark and under visible-light irradiation, respectively. 


$$
\alpha h v=A(h v-E g)^{n / 2}
$$
where $\alpha, h, v$, and $A$ are the absorption coefficient, Planck's constant, light frequency, and a constant, respectively, for direct semiconductors, the value of $\mathrm{n}$ is 1 .

Afterwards, fluorescence tests were performed to research the separation and recombination of photogenerated $\mathrm{e}^{-} / \mathrm{h}^{+}$in the catalysts. As depicted in Fig. 3c, the obtained strong fluorescence emission intensity of HT located at 425 and 575 $\mathrm{nm}$ indicates a faster $\mathrm{e}^{-} \mathrm{h}^{+}$recombination rate, while the fluorescence intensity of HTAx displays a distinct decrease as the $\mathrm{Au}$ content increases, manifesting that the incorporation of $\mathrm{Au}$ nanoparticles alleviates photogeneration carriers recombination, and promotes the transfer of charges. Among them, the weakest intensity of HTA4 implied the highest $e^{-}-h^{+}$ mobility. The above results were further validated by I-t measurements. In Fig. 3d, it can be seen that HTA4 exhibits an enhanced photocurrent response compared to HT. This further supports the significantly improved photoinduced e mobility in HTA4 during light illumination.

EIS were further used to validate the charge carriers transfer behavior of HT and various HTAx photocatalysts (Figs. 3e-f). As shown in Fig. 3e, the semicircle radius at high frequency region of HTA4 is obviously smaller than the others, which reveals a more efficient charge transfer process at the photoelectrode-electrolyte interface, ${ }^{[66]}$ as indicated in the equivalent circuit of HTA4 (the inset of Fig. 3e). The Nyquist plots of HT and HTA4 electrodes under visible-light irradiation and in dark conditions are shown in Fig. 3f. Both HT and HTA4 exhibit a large impedance radius in dark, signifying that the generation and transfer of photo-generated charge carriers are more likely to occur under visible light irradiation, which is more conducive to the subsequent improvement of photocatalytic activity. The above results indicate that the incorporation of the Au nanoparticles into the catalytic system dramatically improves the separation efficiency of photogenerated charges, which will be beneficial to accelerate $\mathrm{H}_{2}$ generation at the interface. The separation mechanism of photogenerated $\mathrm{e}^{-} / \mathrm{h}^{+}$in HT and HTA4 was further confirmed by their band structures.

Fig. 4a displays the Mott-Schottky plots of HT and HTA4 samples in dark at frequency of $1 \mathrm{kHz}$. A positive slope of the line implies a n-type conductivity. The $x$-axis intercept of the curve indicates the flat band potential $\left(\mathrm{V}_{\mathrm{fb}}\right)$ with the values of -0.45 and $-0.92 \mathrm{~V}(v s . \mathrm{Ag} / \mathrm{AgCl}$ at $\mathrm{pH}=6.6)$, corresponding to -0.25 and $-0.72 \mathrm{~V}$ ( $v s$. the normal hydrogen electrode, NHE), for HT and HTA4, respectively. ${ }^{[67]}$ For n-type semiconductors, the $\mathrm{E}_{\mathrm{CB}}$ of HT and HTA4 are -0.25 and $-0.72 \mathrm{~V}$ ( $v$ s. NHE). Considering the $\mathrm{E}_{\mathrm{g}}$ of HT and HTA4 calculated by UV-vis DRS spectra to be 2.92 and $2.68 \mathrm{eV}$ (Fig. 3b), based on the empirical formula $E_{g}=E_{V B}-E_{C B}$, the $E_{V B}$ of HT and HTA4 are +2.67 and $+1.96 \mathrm{~V} v s$. NHE. The energy band structure diagram is shown in Fig. 4b. Compared with HT, the band gap of HTA4 moves in a more negative direction (CB: $-0.25 \rightarrow-$ $0.72 \mathrm{~V}$ ), which is advantageous for the photocatalytic $\mathrm{H}_{2}$ evolution $\left(\mathrm{E}\left(\mathrm{H}^{+} / \mathrm{H}_{2}=0 \mathrm{~V} v s\right.\right.$. NHE $\left.)\right)$.

\subsection{Photocatalytic activity measurement}

The photocatalytic activity of the samples was evaluated with their use in $\mathrm{H}_{2}$ evolution reaction from water splitting and biomass reforming. Fig. 5a reveals a time-dependent cocatalyst-free photocatalytic $\mathrm{H}_{2}$ generation from water over various HTAx photocatalysts using TEOA as a sacrificial reagent under a visible-light irradiation. As a blank experiment, only trace $\mathrm{H}_{2}$ was generated with the use of P25 $(2.73 \mu \mathrm{mol}$ after $240 \mathrm{~min}$ ) because of the rapid recombination of photogenerated charge carriers and the narrow light response scope. In the case of HT, the detected small $\mathrm{H}_{2}$ evolution (7.02 $\mu \mathrm{mol}$ after $240 \mathrm{~min}$ ) was attributable to the doping of $\mathrm{Ti}^{3+}$ and the hierarchical architectures formed by the directional assembly of nanorods, resulting in the proper $\mathrm{CB}$ position and the photogenerated $\mathrm{e}^{-}$with sufficient reduction ability. For HTAx, it is apparent that the amount of $\mathrm{H}_{2}$ production gradually increases as the irradiation time is prolonged. With increasing the content of $\mathrm{Au}$ nanoparticles in samples, $\mathrm{H}_{2}$ production of HTAx increases from HTA1 to HTA4, and then decreases for HTA5. The decreasing of $\mathrm{H}_{2}$ production with
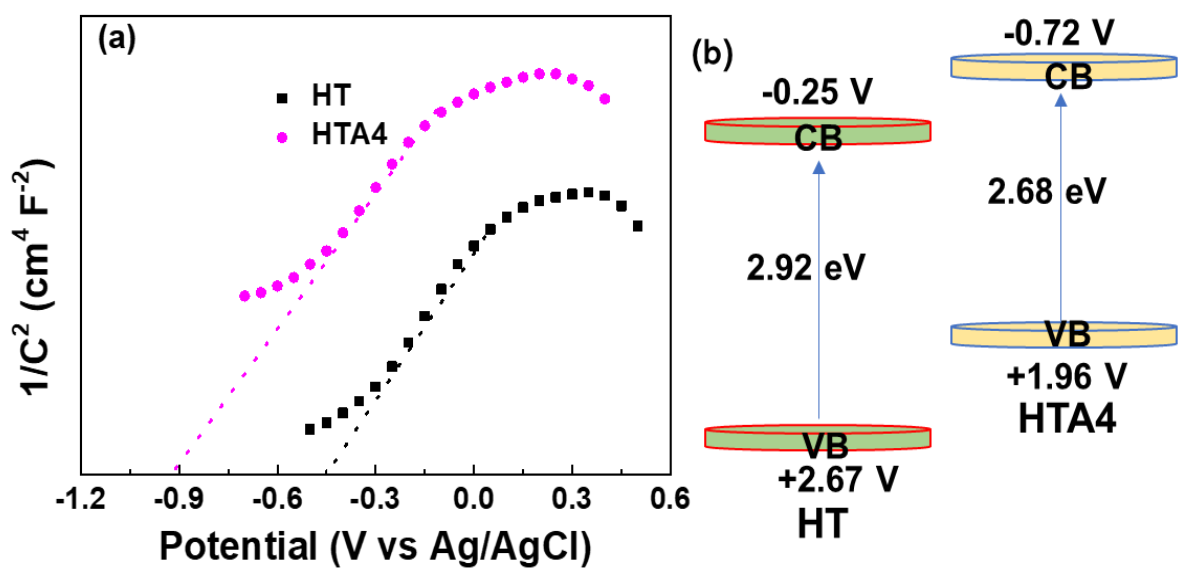

Fig. 4 (a) The Mott-Schottky plots of HT and HTA4 photocatalysts $v s \mathrm{Ag} / \mathrm{AgCl}$ at $\mathrm{pH}=6.6$; (b) Band structure diagrams of HT and HTA4. 
increasing the Au incorporation may be due to the shielding effect and the formation of charge recombination centers. Another aspect may be the accumulation of Au may limit its absorption of light, or the decrease of specific area leading to the reduction of reaction sites for adsorbed reactants, which is consistent with the BET analysis in Fig. 2b. ${ }^{[68]}$ HTA4 exhibited the optimal $\mathrm{H}_{2}$ generation activity with a rate of $349.75 \mu \mathrm{mol}$ $\mathrm{h}^{-1} \mathrm{~g}^{-1}$, which was about 47.52 and 9.89 times higher than that of P25 and HT, respectively, as shown in Fig. 5b. The modification of the catalyst with the incorporation of $\mathrm{Au}$ nanoparticles with a SPR effect dramatically improves the separation efficiency of photogenerated charges and accelerates the $\mathrm{H}_{2}$ generation at the interface. ${ }^{[64,65]}$ HTA4 sample exhibited good stability during the photocatalytic $\mathrm{H}_{2}$ production, and the $\mathrm{H}_{2}$ activity was decreased slightly after five cycles, which might be due to the fact that part of the cocatalysis Au fell off from the HT crystalline nanorods due to the centrifugation in the process of recycling (Fig. $5 \mathrm{c}$ ).

Photocatalytic reforming of biomass is a promising method for the sustainable production of $\mathrm{H}_{2}$. In our study, glucose was used as a simplified model for $\mathrm{H}_{2}$ evolution by photocatalytic reforming. Fig. $5 \mathrm{~d}$ shows the $\mathrm{H}_{2}$ evolution profiles obtained from the photocatalytic glucose reforming over various photocatalysts. Similar to water splitting, there was only small $\mathrm{H}_{2}$ evolved over P25 (3.87 $\mu$ mol after $\left.240 \mathrm{~min}\right)$ and HT (7.59 $\mu \mathrm{mol}$ after $240 \mathrm{~min}$ ) in glucose solution due to its wide band gap and the limited visible light absorption. Compared with P25 and HT, the HTAx catalysts exhibit a remarkably improved $\mathrm{H}_{2}$ production activity by photocatalytic glucose reforming, with HTA4 showing the highest $\mathrm{H}_{2}$ generation rate $\left(616.23 \mu \mathrm{mol} \mathrm{h}^{-1} \mathrm{~g}^{-1}\right)$. The $\mathrm{H}_{2}$ evolution performance of HTA4 was about 46.23 and 15.81 times higher than those of P25 and HT (Fig. 5e). These results manifested that the doping of $\mathrm{Ti}^{3+}$ introduces Ov can effectively accelerate the separation of photogenerated carrier, in addition, the 3D hierarchical architectures formed by the directional assembly of crystalline nanorods, and the SPR effect of the incorporated $\mathrm{Au}$ nanoparticles further dramatically prolonged the life of photogenerated carriers and accelerated the $\mathrm{H}_{2}$ generation at the interface. ${ }^{[63-65]}$ Hydrogen source is very important for biomass reforming. A large number of experimental studies have shown that hydrogen comes from the decomposition of solvent water. Glucose oxidation, while acting as a hole scavenging agent, can also produce some high-value chemicals. ${ }^{[14,69]}$ Considering the glucose is not a typical biomass, photocatalytic biomass reforming for $\mathrm{H}_{2}$ generation was then expanded to other biomass, such as fructose, xylose, galactose, saccharose, xylan, and cellobiose (Fig. 6a). Glucose, fructose, and galactose with smaller substrate size had higher $\mathrm{H}_{2}$ production rate (Fig. $6 \mathrm{~b}$ ).
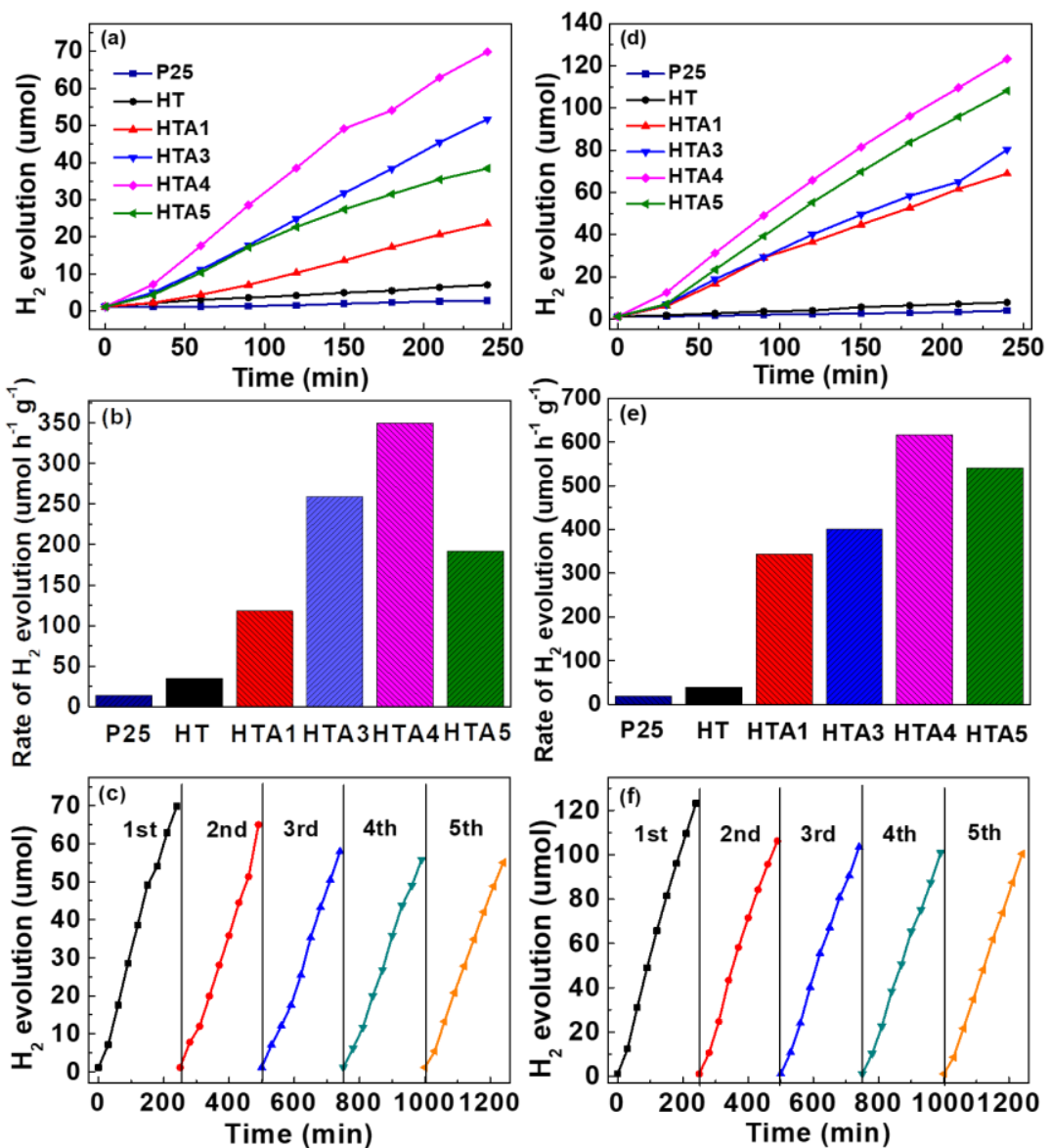

Fig. 5 Time-dependent photocatalytic $\mathrm{H}_{2}$ generation from (a) water and (d) photocatalytic reforming biomass for different photocatalysts; Photocatalytic $\mathrm{H}_{2}$ generation rate from (b) water and (e) photocatalytic reforming biomass for different photocatalysts; Cycling stability tests of $\mathrm{H}_{2}$ generation from (c) water and (e) photocatalytic reforming biomass over HTA4. 


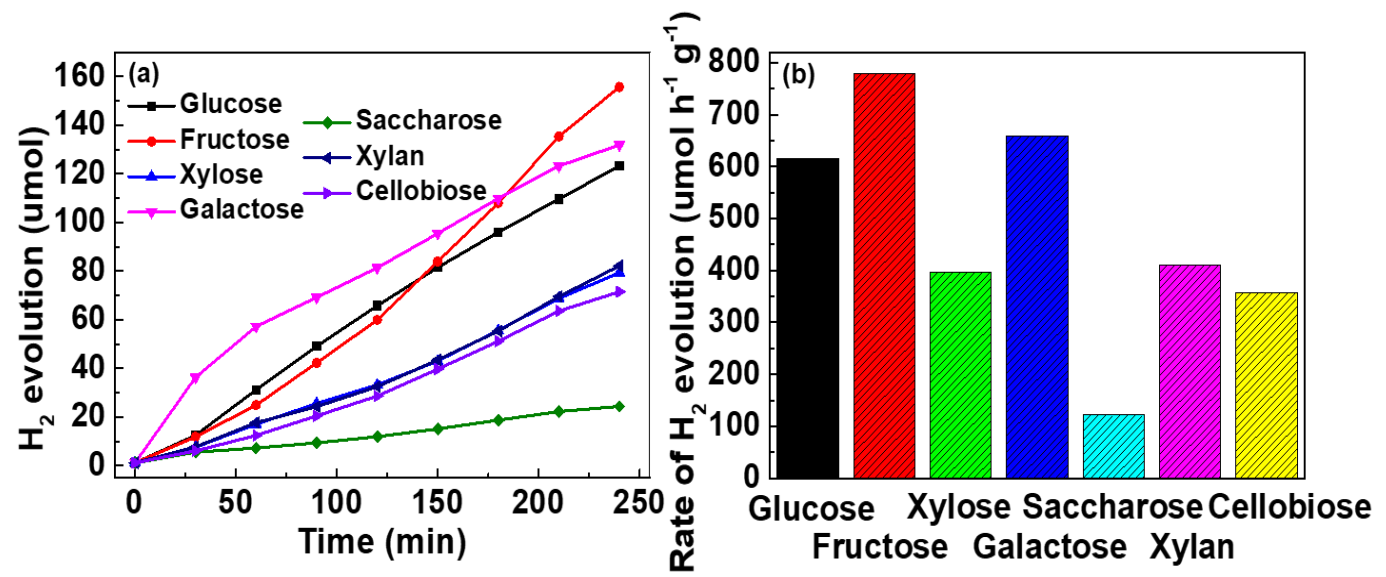

Fig. 6 (a) Time-dependent photocatalytic $\mathrm{H}_{2}$ generation and (b) Photocatalytic $\mathrm{H}_{2}$ generation rate from different biomass, such as glucose, fructose, xylose, galactose, saccharose, xylan, and cellobiose.

The excellent photocatalytic performance of HTA4 is compared in Fig. 7 with other $\mathrm{TiO}_{2}$-based catalysts reported in the literatures. Good activity is observed in HTA4 under a lower reaction temperature without the need of additional $\mathrm{Pt}$ co-catalyst. It is worth noting that the use of natural renewable biomass as $\mathrm{e}^{-}$donor avoids the waste of valuable chemicals, such as alcohols, organic acids, or amines. It also provides a valuable reference for our subsequent biomass reforming to produce $\mathrm{H}_{2}$ and the preparation of platform chemicals. In addition, after 5 consecutive cycles $(20 \mathrm{~h})$ of HTA4 stability experiments (Fig. 5f), the $\mathrm{H}_{2}$ production rate was decreased slightly, which might be due to the coverage of active sites for $\mathrm{H}_{2}$ production by liquid by-products in the biomass reforming process besides the partial co-catalyst abscissions as mentioned above. Moreover, the PXRD and TEM characterizations of the recovered photocatalyst, as shown in Fig. 8, demonstrate no structural nor morphological change, which supporting the excellent stability in a long-term photocatalytic reaction. The above results indicate that the photocatalytic $\mathrm{H}_{2}$ production is feasible in the absence of $\mathrm{Pt}$ co-catalyst, especially in the process of photocatalytic biomass reforming (where glucose is also used as a hole scavenger). This offers a very powerful alternative among the practical and low-cost technologies in the $\mathrm{H}_{2}$-based energy systems.

The potential $\mathrm{H}_{2}$ generation mechanism photocatalysis over HTA4 is explained in Fig. 9. As evidenced in Figs. 3 and 5, The $\mathrm{Ti}^{3+}$ doping introduces Ov can form a new sublevel state at the near bottom of the HT CB, which can shorten the band gap of HT, and thus speed up the $\mathrm{e}^{-}-\mathrm{h}^{+}$ separation, then prominently improve the photo-response for visible light, meanwhile, the 3D hierarchical nanorods architectures, and the incorporation of Au nanoparticles cause HTA4 with an enhanced visible light absorption and hindered the recombination of the photogenerated $\mathrm{e}^{-} / \mathrm{h}^{+}$pairs. This combination of factors makes HTA4 as the best photocatalyst in $\mathrm{H}_{2}$ evolution reaction among the studied samples at low temperature without any additional $\mathrm{Pt}$ co-catalyst. Under visible light irradiation, HT became active to generate $\mathrm{e}^{-} / \mathrm{h}^{+}$, the $\mathrm{e}^{-}$in the CB of HT migrated to Au nanoparticles motivated by the Schottky barrier, which improved the separation efficiency of photogenerated charge carriers. The VB position of HTA4 was more positive than the standard redox potential of $\mathrm{OH}^{-} \% \mathrm{OH}$, indicating the $\cdot \mathrm{OH}$ can be generated for the following glucose oxidation. And the $\mathrm{h}^{+}$remaining in the VB of HT reacted with sacrificial reagent (TEOA) in the aqueous solution for photocatalytic $\mathrm{H}_{2}$ evolution from water splitting. Since the CB position of HTA4 $(-0.72 \mathrm{~V}$, Fig. $4 \mathrm{~b})$ is more negative than the reduction potential of $\mathrm{H}^{+} / \mathrm{H}_{2}(0 \mathrm{~V} v s$. NHE at $\mathrm{pH}=7$ ), the accumulated $\mathrm{e}^{-}$on $\mathrm{Au}$ nanoparticles could react by with $\mathrm{H}^{+}$to form $\mathrm{H}_{2}$ (Fig. 9a). For photocatalytic $\mathrm{H}_{2}$ evolution from
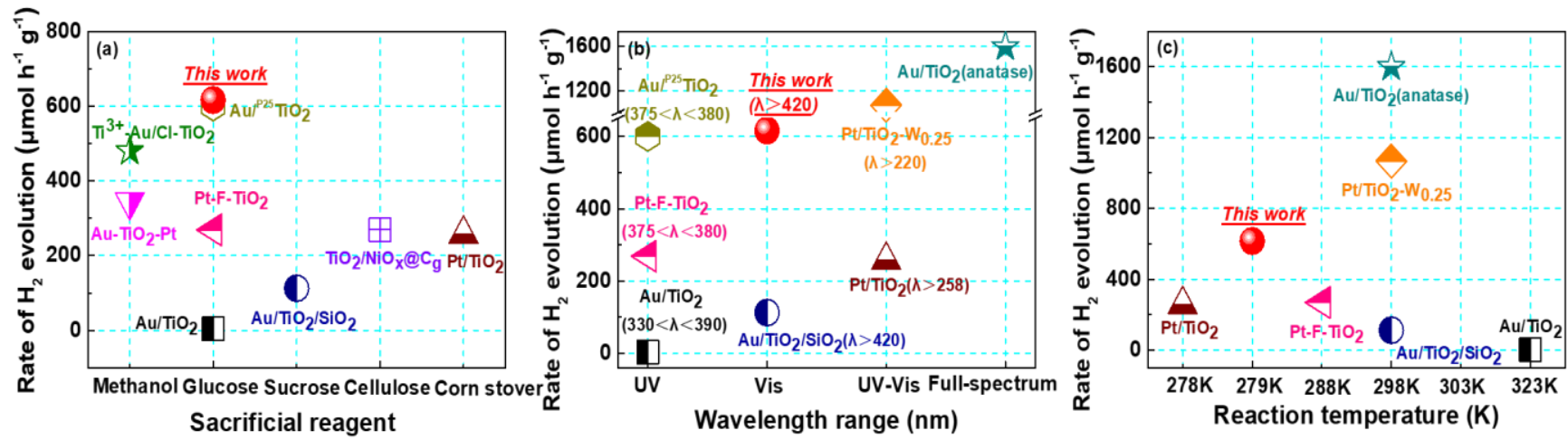

Fig. 7 Comparison of the $\mathrm{H}_{2}$ generation activity for some $\mathrm{TiO}_{2}$-based photocatalysts under different (a) wavelength range, (b) sacrificial reagent and (b) reaction temperature (All details were described in Table S1 in the Supporting Information). 

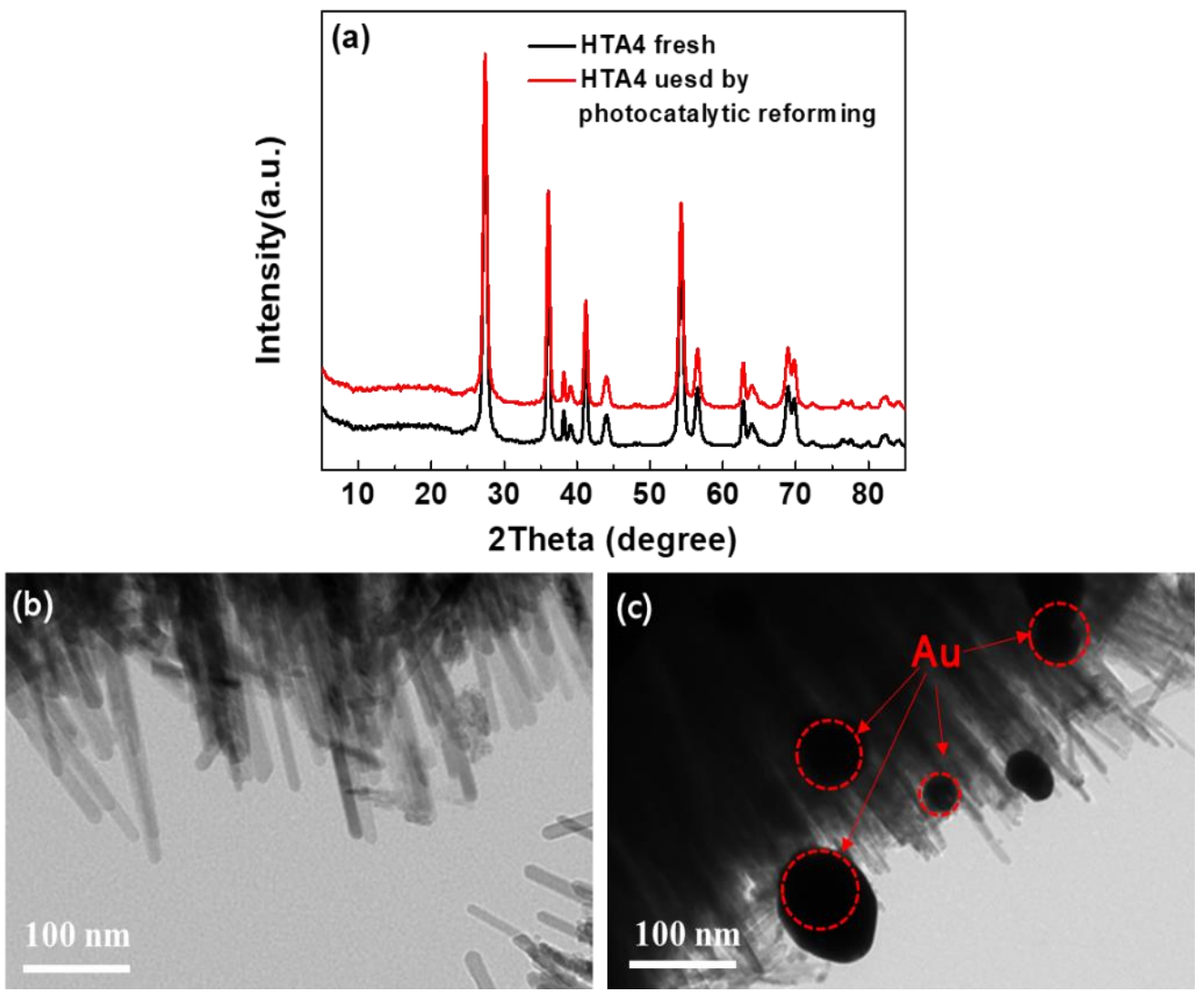

Fig. 8 (a) PXRD patterns of HTA4 photocatalyst before and after the photocatalytic glucose reforming reaction; the TEM of (b) HT and (c) HTA4 photocatalyst after the photocatalytic reaction.

glucose reforming, the positively charged $\mathrm{h}^{+}$and $\cdot \mathrm{OH}$ with a strong oxidizing ability in the VB of HTA4 would participate in the glucose reforming process, which lead to the production of more $\mathrm{e}^{-}$that contribute to the photocatalytic $\mathrm{H}_{2}$ generation on the surface of $\mathrm{Au}$ nanoparticles. Meanwhile, as shown in Fig. 9b, arabinose, formic acid, and erythrose are the most possible products obtained during the process of glucose oxidization. ${ }^{[35,70]}$

\section{Conclusions}

A simple one-step solvothermal approach has been successfully carried out to prepare $\mathrm{Au} /$ hierarchical $\mathrm{TiO}_{2}$ architectures as photocatalysts through the assembly under mild conditions of crystalline nanorods with different amounts of gold. Among the prepared materials, HTA4 exhibits the prominent photocatalytic $\mathrm{H}_{2}$ generation performance from glucose reforming at low temperature without any additional $\mathrm{Pt}$ co-catalyst, reaching a $\mathrm{H}_{2}$ evolution rate that is about 46.23 and 15.81 times higher than that of P25 and HT, respectively. The prominent photocatalytic $\mathrm{H}_{2}$ generation performance was ascribed to the particular hierarchical $\mathrm{TiO}_{2}$ architectures, which improved the light capturing capability, restrained the recombination of photogenerated carriers, and the SPR effect of Au nanoparticles that further enhanced the separation

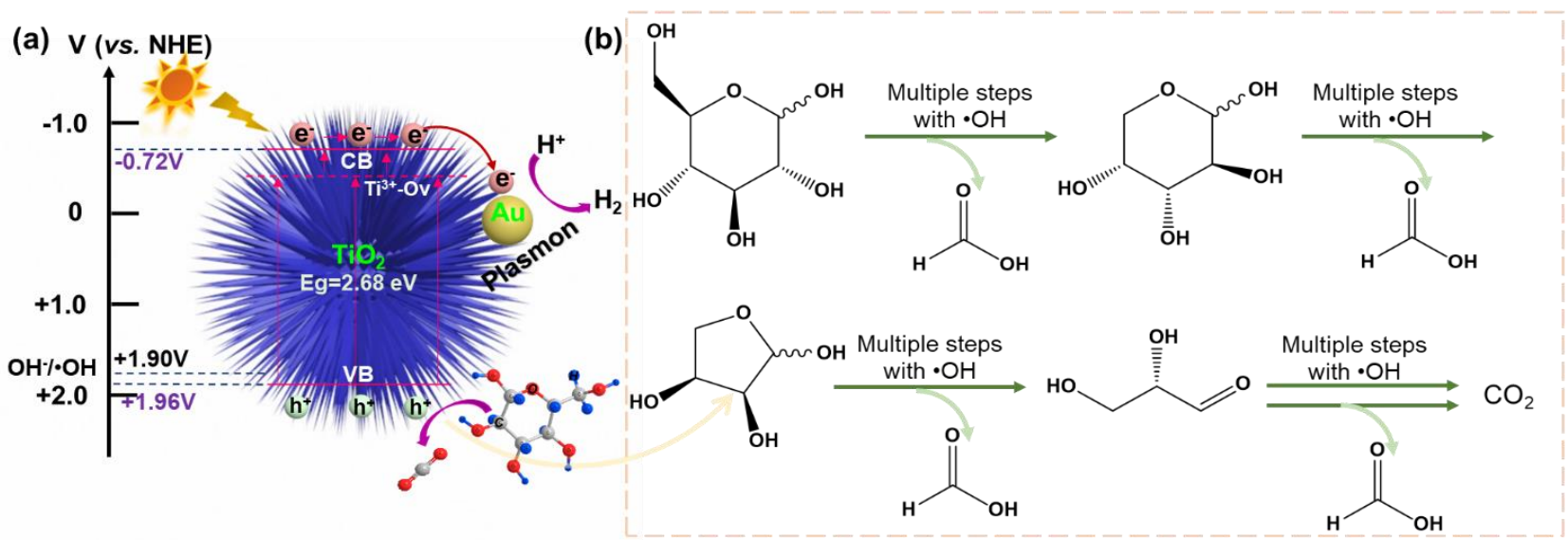

Fig. 9 (a) Illustration of $\mathrm{H}_{2}$ generation mechanisms and (b) the possible reactions of glucose by photocatalysis over HTA4 under visible light irradiation. 
efficiency of photogenerated charges and extended the visible light absorption. In the process of photocatalytic glucose reforming for $\mathrm{H}_{2}$ evolution, the $\mathrm{e}^{-}$in $\mathrm{CB}$ reduced the proton of water to generate $\mathrm{H}_{2}$, meanwhile, glucose was oxidized by $\mathrm{h}^{+}$, further promoting the $\mathrm{H}_{2}$ evolution. This work demonstrates promising results to sustainably produce $\mathrm{H}_{2}$ from environmentally renewable biomass by using solar energy, which is worthy of reference and promotion for the sustainable development of human beings.

\section{Acknowledgements}

This work was supported by the National Natural Science Foundation of China (No. 32071713), the National Innovation Training Program of Northeast Forestry University (No. 202010225069), the Outstanding Youth Foundation Project of Heilongjiang Province (No. JQ2019C001), and the Central University Basic Scientific Research Project of China (No. 2572020DX01).

\section{Conflict of interest}

There are no conflicts to declare.

\section{Supporting information}

Applicable.

\section{References}

[1] X. Chen, S. Shen, L. Guo, S. S. Mao, Chem. Rev., 2010, 110, 6503-6570, doi: 10.1021/cr1001645.

[2] S. Wang, B. Zhu, M. Liu, L. Zhang, J. Yu, M. Zhou, Appl. Catal. B, 2019, 243, 19-26, doi: 10.1016/j.apcatb.2018.10.019.

[3] Y. Zhou, S. Wu, Y. Ma, H. Zhang, X. Zeng, F. Wu, F. Liu, J. E. Ryu, Z. Guo, ES Energ. Environ., 2020, 9, 28-40, doi: 10.30919/esee8c150.

[4] G. Liu, G. Yao, J. Xu, X. Yan, ES Energ. Environ., 2020, 9, 41-49, doi: 10.30919/esee8c450.

[5] S. Zhao, H.Wang, ES Energ. Environ., 2020, 9, 59-66, doi: $10.30919 /$ esee 8 c262.

[6] S. D. Satpute, J. S. Jagtap, P. K. Bhujbal, S. M. Sonar, P. K. Baviskar, S. R. Jadker, H. M. Pathan, ES Energ. Environ., 2020 , 9, 89-94, doi: 10.30919/esee8c720.

[7] D. Li, J. Sun, R. Ma, J. Wei, ES Energ. Environ., 2020, 9, 8288, doi: 10.30919/esee8c722.

[8] P. Vairale, V. Sharma, B. Bade, A. Waghmare, P. Shinde, A. Punde, V. Doiphode, R. A. Aher, S. Pandharkar, Eng. Sci., 2020, 11, 76-84, doi: 10.30919/es8d0023.

[9] Z. Caia, P. Wanga, J. Yanga, X. Wanga, ES Energ. Environ., 2019, 5, 22-36, doi: 10.30919/esee8c320.

[10] J. D. Desai, P. K. Baviskar, K. N. Hui, H. M. Pathan, ES Energ. Environ., 2018, 2, 21-30, doi: 10.30919/esee8c211.

[11] H. Borate, A. Bhorde, A. Waghmare, S. Nair, S. Pandharkar, A. Punde, P. Shinde, P. Vairale, V. Jadkar, R. G. Waykar, S. Rondiya, Y. Hase, ES Mater. Manuf., 2021, 11, 30-39, doi: 10.30919/esmm5f934.

[12] B. Liu, Y. Jin, G. Xie, ES Energ. Environ., 2018, 1, 56-66, doi: 10.30919/esee8c160.

[13] J. C. Colmenares, R. S. Varma, V. Nair, Chem. Soc. Rev., 2017, 46, 6675-6686, doi: 10.1039/c7cs00257b.

[14] D. W. Wakerley, M. F. Kuehnel, K. L. Orchard, K. H. Ly, T. E. Rosser, E. Reisner, Nat. Energy, 2017, 2, 17021, doi: 10.1038/nenergy.2017.21.

[15] T. Wei, Z. Jin, F. Li, D. Yan, L. Xu, Photoch. Photobio. Sci., 2020, 19, 80-87, doi: 10.1039/C9PP00427K.

[16] L. Wei, K. Lozano, Y. Mao, Eng. Sci., 2018, 3, 62-66, doi: 10.30919/es8d723.

[17] Q. Yuan, R. Wang, Q. Wang, P. Sun, R. Nie, X. Wang, ES Mater. Manuf. 2018, 2, 9-15, doi: 10.30919/esmm5f112.

[18] H. Yu, L. Yang, D. Cheng, D. Cao, Eng. Sci., 2018, 3, 54-61, doi: 10.30919/es8d729.

[19] P. Yang, H. Zhao, Y. Yang, P. Zhao, X. Zhao, L.Yang, ES Mater. Manuf., 2020, 7, 34-39, doi: 10.30919/esmm5f618.

[20] Q. F. Liu, Q. Zhang, B. R. Liu, S. Li, J. J. Ma, Chinese J. Catal., 2018, 39, 542-548, doi: 10.1016/S1872-2067(18)63044$\mathrm{X}$.

[21] K. Qi, W. Lv, I. Khan, S. Y. Liu, Chinese J. Catal., 2020, 41, 114-121, doi: 10.1016/S1872-2067(19)63459-5.

[22] A. Wu, C. Tian, Y. Jiao, Q. Yan, G. Yang, H. Fu, Appl. Catal. $B, 2017$, 203, 955-963, doi: 10.1016/j.apcatb.2016.11.009.

[23] X. Yu, X. Fan, L. An, G. Liu, Z. Li, J. Liu, P. Hu, Carbon, 2018, 128, 21-30, doi: 10.1016/j.carbon.2017.11.078.

[24] X. Li, K. Chen, X. Ji, X. Yuan, Z. Lei, M. W. Ullah, J. Xiao, G. Yang, Eng. Sci., 2021, 13, 106-120, doi: 10.30919/es8d1159.

[25] X. Wang, X. Zeng, D. Cao, Eng. Sci., 2018, 1, 55-63, doi: $10.30919 /$ es. 180325 .

[26] J. Cai, J. Tian, H. Gu, Z. Guo, ES Mater. Manuf., 2019, 6, 68-74, doi: 10.30919/esmm5f611.

[27] B. Yuan, L. Li, V. Murugadoss, S. Vupputuri, J. Wang, N. Alikhani, Z. Guo, ES Food Agroforestry., 2020, 1, 41-52, doi: 10.30919/esfaf0004.

[28] J. Cai, W. Xu, Y. H. Liu, Z. Z. Zhu, G. Liu, W. P. Ding, G. Z. Wang, H. B. Wang, Y. C. Luo, Eng. Sci., 2019, 5, 21-29, doi: 10.30919/es8d669.

[29] B. O. O. Boni, L. Lamboni, B. M. Bakadia, S. A. Hussein, G. Yang, Eng. Sci., 2020, 10, 68-77, doi: 10.30919/es8d906.

[30] S. Xie, X. Zhang, M. P. Walcott, H. Lin, Eng. Sci., 2018, 2, 4-16, doi: 10.30919/es.1803302.

[31] G. Iervolino, V. Vaiano, J. J. Murcia, L. Rizzo, G. Ventre, G. Pepe, P. Campiglia, M. C. Hidalgo, J. A. Navío, D. Sannino, J. Catal., 2016, 339, 47-56, doi: 10.1016/j.jcat.2016.03.032.

[32] X. Xu, J. Zhang, S. Wang, Z. Yao, H. Wu, L. Shi, Y. Yin, S. Wang, H. Sun, J. Colloid Interf. Sci., 2019, 555, 22-30, doi: 10.1016/j.jcis.2019.07.066. 
[33] M. F. Kuehnel, E. Reisner, Angew. Chem. Int. Edit., 2018, 57, 3290-3296, doi: 10.1002/ange.201710133.

[34] C. G. Silva, M. J. Sampaio, R. R. N. Marques, L. A. Ferreira, P. B. Tavares, A. M. T. Silva, J. L. Faria, Appl. Catal. B, 2015, 178, 82-90, doi: 10.1016/j.apcatb.2014.10.032.

[35] P. She, J. Qin, H. Rao, B. Guan, J. Yu, Mater. Chem. Front., 2020, 4, 1671-1678, doi: 10.1016/j.jcat.2014.03.009.

[36] C. Ma, Y. Li, H. Zhang, Y. Chen, C. Lu, J. Wang, Chem. Eng. J., 2015, 273, 277-285, doi: 10.1016/j.cej.2015.03.090.

[37] L. Zhang, W. Wang, S. Zeng, Y. Su, H. Hao, Green Chem., 2018, 20, 3008-3013, doi: 10.1039/C8GC01398E.

[38] M. Yu, T. Yu, S. Chen, Z. Guo, I.Seok, ES Mater. Manuf., 2020, 7, 64-69, doi: 10.30919/esmm5f712.

[39] B. Wang, K. Wei, X. Mo, J. Hu, G. He, Y. Wang, W. Li, Q. He, ES Mater. Manuf., 2019, 4, 51-57, doi: 10.30919/esmm5f215. [40] Y. Zhao, Q. Zeng, T. Feng, C. Xia, C. Liu, F. Yang, K. Zhang, B. Yang, Mater. Chem. Front., 2019, 3, 2659-2667, doi: 10.1039/C9QM00556K.

[41] Y. Li, S. Yang, Z. Liang, Y. Xue, H. Cui, J. Tian, Mater. Chem. Front., 2019, 3, 2673-2680, doi: 10.30919/esmm5f109.

[42] C. Shi, H. Qi, Z. Sun, K. Qu, Z. Huang, J. Li, M. Dong, Z. Guo, J. Mater. Chem. C, 2020, 8, 2238-2247, doi: 10.1039/C9TC05513D.

[43] S. Zeng, H. Sun, Y. Wu, Y. Shang, P. She, Q. He, S. Yin, Z. Liu, Inorg. Chem. Front., 2018, 5, 626-634, doi: 10.1039/C7QI00717E.

[44] X. Sun, S. Xu, Y. Gao, M. Yue, Q. Yue, B. Gao, CrystEngComm, 2017, 19, 2187-2194, doi: 10.1039/C7CE00080D.

[45] S. Xu, X. Sun, Y. Zhao, Y. Gao, Y. Wang, Q. Yue, B. Gao, Appl. Surf. Sci., 2018, 448, 78-87, doi: 10.1016/j.apsusc.2018.04.100.

[46] S. Sadhu, P. Gupta, P. Poddar, ACS Appl. Mater. Inter., 2017, 9, 11202-11211, doi: 10.1021/acsami.6b15420.

[47] B. Gupta, A. A. Melvin, T. Matthews, S. Dash, A. K. Tyagi, Renew. Sust. Energ. Rev., 2016, 58, 1366-1375, doi: 10.1016/j.rser.2015.12.236.

[48] H. Chen, L. Shao, Q. Li, J. Wang, Chem. Soc. Rev., 2013, 42, 2679-2724, doi: 10.1039/c2cs35367a.

[49] S. Veziroglu, A. L. Obermann, M. Ullrich, M. Hussain, M. Kamp, L. Kienle, T. Leissner, H. G. Rubahn, O. Polonskyi, T. Strunskus, J. Fiutowski, M. E. Souni, J. Adam, F. Faupel, O. C. Aktas, ACS Appl. Mater. Inter., 2020, 12, 14983-14992, doi: 10.1021/acsami.9b18817.

[50] W. Jiao, L. Wang, G. Liu, G. Q. Lu, H. M. Cheng, ACS Catal., 2012, 2, 1854-1859, doi: 10.1021/cs300229e.

[51] T. Lan, T. Wang, W. Zhang, N. L. Wu, M. Wei, J. Alloy. Compd., 2017, 699, 455-462, doi: 10.1016/j.jallcom.2016.12.337.
[52] L. Xiang, X. Zhao, J. Yin, B. Fan, J. Mater. Sci., 2011, 47, 1436-1445, doi: 10.1007/s10853-011-5924-7.

[53] R. Raji, K. G. Gopchandran, J. Hazard. Mater, 2019, 368, 345-357, doi: 10.1016/j.jhazmat.2019.01.052.

[54] Y. Zhou, C. Chen, N. Wang, Y. Li, H. Ding, J. Phys. Chem. C, 2016, 120, 6116-6124, doi: 10.1021/acs.jpcc.6b00655.

[55] E. Bae, T. Ohno, Appl. Catal. B, 2009, 91, 634-639, doi: 10.1016/j.apcatb.2009.06.034.

[56] X. Yu, X. Fan, Z. Li, J. Liu, Dalton Trans., 2017, 46, 11898 11904, doi: 10.1039/c7dt02824e.

[57] H. Yu, Y. Zhao, C. Zhou, L. Shang, Y. Peng, Y. Cao, L. Z. Wu, C. H. Tung, T. Zhang, J. Mater. Chem. A, 2014, 2, 3344-3351, doi: 10.1039/C3TA14108J.

[58] D. Kandi, A. Behera, S. Martha, B. Naik, K. M. Parida, J. Environ. Eng., 2019, 7, 102821, doi: 10.1016/j.jece.2018.102821. [59] S. Tan, Z. Xing, J. Zhang, Z. Li, X. Wu, J. Cui, J. Kuang, Q. Zhu, W. Zhou, J. Catal., 2018, 357, 90-99, doi: 10.1016/j.jcat.2017.08.006.

[60] J. Li, H. C. Zeng, Chem. Mater., 2006, 18, 4270-4277, doi: $10.1021 / \mathrm{cm} 060362 \mathrm{r}$.

[61] T. Han, H. Wang, X. Zheng, RSC Adv., 2016, 6, 7829-7837, doi: 10.1039/C5RA25337C.

[62] J. Yang, D. Wang, H. X. Han, C. Li, Accounts Chem. Res., 2013, 46,1900-1909, doi: 10.1021/ar300227e.

[63] F. Zuo, L. Wang, T. Wu, Z. Zhang, D. Borchardt, P. Feng, J. Am. Chem. Soc., 2010, 132, 11856-11857, doi: 10.1002/chin.201049012.

[64] Z. G. Sun, X. S. Li, J. L. Liu, Y. C. Li, B. Zhu, A. M. Zhu, J. Catal., 2019, 375, 380-388, doi: 10.1016/j.jcat.2019.05.027.

[65] V. Vinesh, A. R. M. Shaheer, B. Neppolian, Ultrason. Sonochem., 2019, 50, 302-310, doi: 10.1016/j.ultsonch.2018.09.030.

[66] Y. Li, Y. Zhou, Y. Wang, R. Zhou, Q. Ling, H. Niu, W. Zhang, C. Wang, J. Qiu, Z. Guo, J. Xu, Electrochim. Acta, 2019, 293, 230-239, doi: 10.1016/j.electacta.2018.10.035.

[67] Y. Bai, L. Ye, T. Chen, L. Wang, X. Shi, X. Zhang, D. Chen, ACS Appl. Mater. Inter., 2016, 8, 27661-27668, doi: 10.1021/acsami.6b08129.

[68] M. Tahir, M. Siraj, B. Tahir, M. Umer, H. Alias, N. Othman, Appl. Surf. Sci., 2020, 503, 144344.1-144344.12, doi: 10.1016/j.apsusc.2019.144344.

[69] H. Kasap, D. S. Achilleos, A. Huang, E. Reisner, J. Am. Chem. Soc., 2018, 140, 11604-11607, doi: 10.1021/jacs.8b07853. [70] M. Bellardita, E. I. García-López, G. Marcì, G. Nasillo, L. Palmisano, Eur. J. Inorg. Chem., 2018, 41, 4522-4532, doi: 10.1002/ejic. 201800663 . 


\section{Author information}

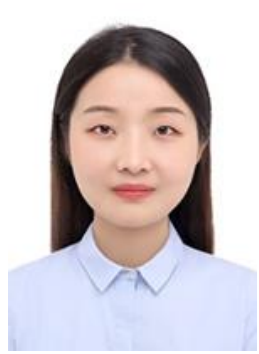

Cai Shi is currently the $3^{\text {rd }}$ year Ph.D. student in Materials Science and Engineering College, Northeast Forestry University. She received her master's degree in 2018 form Northeast Forestry University. Her current research focus on photocatalytic degration of pollutions and photocatalytic biomass reforming for $\mathrm{H}_{2}$ production and high value-added chemicals.

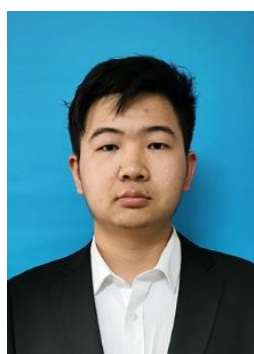

Weijie Yuan is an undergraduate study in Material Science and Engineering College, Northeast Forestry University. He majored in Forest Chemical Industry at Northeast Forestry University in 2018. His current research focus on photocatalytic biomass reforming for $\mathrm{H}_{2}$ production and the application of $\mathrm{TiO}_{2}$ compounds as catalysts.

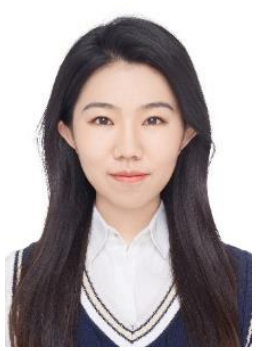

Keqi Qu is currently a third year Ph.D. student in the School of Material at Northeast Forestry University. She received her B.E. degree in forest products chemicals from Northeast Forestry University in 2014. Her current research focuses on the research of supercapacitors and biomass carbon materials.

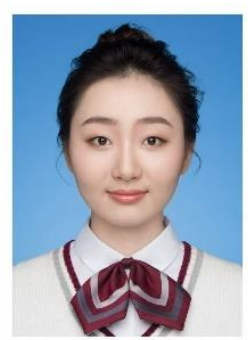

Junming Shi is a Ph.D. study in Material Science and Engineering College, Northeast Forestry University. She received her B.E. degree in forest chemical industry from Northeast Forestry University in 2020. Her current research includes the application of $\mathrm{CN}$ compounds as catalysts and photocatalytic reforming of biomass.

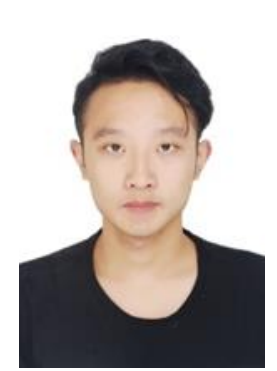

Malin Eqi received his BS degree in 2020 from Sichuan Normal University . He is currently a graduate student in Huang-lab at Northeast Forestry University. His research is centered on photocatalysis and preparation of MOFbased nano-composites.

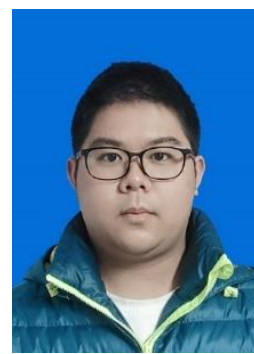

Tan Xushen is an undergraduate study in Material Science and Engineering College, Northeast Forestry University. He majored in Forest Chemical Industry at Northeast Forestry University in 2018. His current research includes the application of $\mathrm{TiO}_{2}$ compounds as photocatalysts and photocatalytic reforming of biomass.

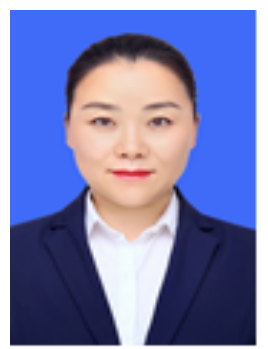

Zhanhua Huang is a professor of the Key laboratory of Bio-based Material Science and Technology of Ministry of Education at Northeast Forestry University, China. She received her B.A. in chemical from Northeast Forestry University of China in 1999, and her M.S. and Ph.D. in forest product chemical processing from Northeast Forestry University of China in 2003 and 2006, respectively. She started as an assistant professor at Northeast Forestry University in 2006, and was promoted to an associate professor and a professor in 2009 and 2014, respectively. Her research interests include functional biomaterial adsorbents, green conversion of sustainable and renewable biomass, novel biomass-based photocatalytic materials and cellulose-based flexible electronics.

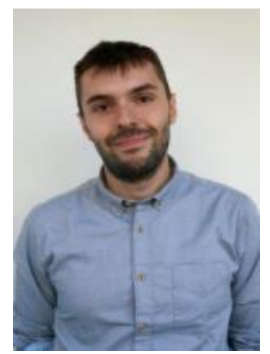

Felipe Gándara is a Tenured Scientist in Materials Chemistry, Materials Science Institute of Madrid. His current research is focused on the improvement of catalytic, conducting and sorption properties of reticular materials through the rational choice of the chemical constituents of the secondary building units. During my career I have participated in 17 research projects funded by public agencies, as well as by private companies, and I have supervised over 15 students and post-docs, and obtained funding as principal investigator in five competitive calls.

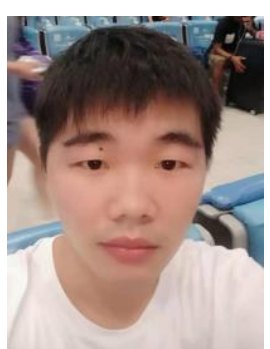

Duo Pan, currently a Ph.D. candidate in National Engineering Research Center for Advanced Polymer Processing Technology at Zhengzhou University, China, he obtained a degree of Master of Science in Shandong University of Science and Technology (2019). His research interest focuses on the preparation of functional 
polymer materials and nanocomposites, as well as applications in catalytic degradation and thermal conduction.

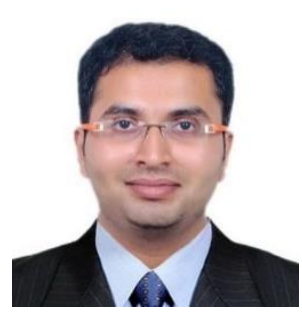

Nithesh Naik is a faculty in the Department of Mechanical and Manufacturing Engineering, Manipal Institute of Technology. He has four years of industry experience in the field of planning and design of HVAC systems. He is a master graduate in Design Engineering from prestigious university Manipal Academy of Higher Education (Institute of Eminence). His research interest includes the development of FE analysis of dental sciences, artificial intelligence, composite materials and design and product development techniques. He has applied 4 patents at Indian Patent Office. He has published several research publications in international journals of repute and has keen interests in medical innovations. He received the award of South India's most exciting young teacher. His areas of expertise include FE analysis of dental and medical sciences, composite materials, design and product development, medical devices and innovations, artificial intelligence, medical web and app development.

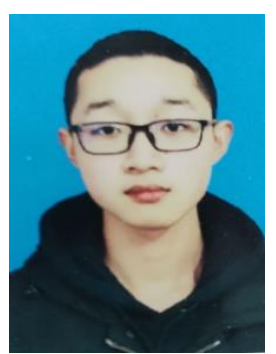

Yixiang Zhang is currently the 3rd year undergraduate student in Material Science and Engineering College, Northeast Forestry. He majored in Forset Chemical Industry at Northeast Forestry University in 2018. His research field is the $\mathrm{TiO}_{2}$ catalysts, and the compounds have potential applications on many fields.

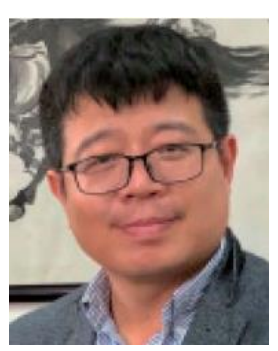

Zhanhu Guo is an Associate Professor in the Department of Chemical and Biomolecular Engineering, the University of Tennessee, Knoxville, USA. He obtained a PhD degree in Chemical Engineering from Louisiana State University (2005), and then received three-year (2005-2008) postdoctoral training from the Mechanical and Aerospace Engineering Department at the University of California Los Angeles. Dr Guo chaired the Composite Division of the American Institute of Chemical Engineers (AIChE, 2010-2011). Dr Guo is the director of the Integrated Composites Laboratory. His current research focuses on multifunctional nanocomposites for energy, electronic and environmental applications.

Publisher's Note: Engineered Science Publisher remains neutral with regard to jurisdictional claims in published maps and institutional affiliations. 